An alternative derivation of the gravitomagnetic clock effect

This article has been downloaded from IOPscience. Please scroll down to see the full text article.

2002 Class. Quantum Grav. 1939

(http://iopscience.iop.org/0264-9381/19/1/303)

View the table of contents for this issue, or go to the journal homepage for more

Download details:

IP Address: 128.206.162.204

The article was downloaded on 23/09/2010 at 20:55

Please note that terms and conditions apply. 


\title{
An alternative derivation of the gravitomagnetic clock effect
}

\author{
Lorenzo Iorio $^{1}$, Herbert Lichtenegger ${ }^{2}$ and Bahram Mashhoon ${ }^{3}$ \\ ${ }^{1}$ Dipartimento di Fisica dell' Università di Bari, via Amendola 173, 70126, Bari, Italy \\ ${ }^{2}$ Institut für Weltraumforschung, Österreichische Akademie der Wissenschaften, \\ A-8042 Graz, Austria \\ ${ }^{3}$ Department of Physics and Astronomy, University of Missouri-Columbia, Columbia, \\ MO 65211, USA
}

Received 10 July 2001, in final form 13 November 2001

Published 13 December 2001

Online at stacks.iop.org/CQG/19/39

\begin{abstract}
The possibility of detecting the gravitomagnetic clock effect using artificial Earth satellites provides the incentive to develop a more intuitive approach to its derivation. We first consider two test electric charges moving on the same circular orbit but in opposite directions in orthogonal electric and magnetic fields and show that the particles take different times in describing a full orbit. The expression for the time difference is completely analogous to that of the general relativistic gravitomagnetic clock effect in the weakfield and slow-motion approximation. The latter is obtained by considering the gravitomagnetic force as a small classical non-central perturbation of the main central Newtonian monopole force. A general expression for the clock effect is given for a spherical orbit with an arbitrary inclination angle. This formula differs from the result of the general relativistic calculations by terms of order $c^{-4}$.
\end{abstract}

PACS number: 0480

\section{Introduction}

The general relativistic clock effect, as worked out in $[1,2]$, is the difference in the orbital periods of two clocks moving in opposite directions along a circular equatorial orbit around a central rotating mass. It is not an easy task to derive the general effect within the framework of Einstein's theory of gravitation; in fact, for orbits of arbitrary inclination to the equatorial plane, the clock effect has been derived only for the case that the orbiting bodies describe spherical orbits of constant 'radius' [2]. Moreover, the case of elliptical orbits has not yet been investigated.

The purpose of this paper is to provide an alternative derivation of this effect for orbits with zero eccentricity, in order to make its comprehension more intuitive by stressing and elucidating 
the analogies and differences between the weak-field and slow-motion approximation of general relativity and electromagnetism. General expressions are given for arbitrary values of inclination as well. They are useful in view of the recent efforts devoted to exploring the possibility of measuring the clock effect by means of artificial near-Earth satellites [3-7].

The paper is organized as follows: in section 2 we deal with an electromagnetic system consisting of two test charges orbiting in opposite directions, acted upon by a central electric field and by a weaker orthogonal magnetic field. The latter is treated perturbatively to first order. Radiative and $\mathcal{O}\left(v^{2} / c^{2}\right)$ effects in such a system are neglected in order to outline the main features common to the gravitational case that is treated in section 3 . In this paper the gravitomagnetic force is viewed classically as a non-central small linear perturbation of the main central Newtonian gravitoelectric monopole force. Section 5 contains concluding remarks.

\section{The electromagnetic scenario}

Let us consider two identical point charges $q$ of mass $m$ orbiting a central spherically symmetric distribution of total charge $Q$ of opposite signs, e.g. $Q<0$ and $q>0$. We suppose that the two charges follow identical but opposite closed circular orbits and denote the speeds of the counterclockwise and clockwise moving charges by $v_{+}$and $v_{-}$, respectively, i.e. we assume the counterclockwise direction to be positive. In cylindrical coordinates $\{\rho, \phi\}$, the equation of motion of the two charges reads

$$
m \frac{v_{ \pm}^{2}}{\rho}=|q| E
$$

with $E=|Q| / \rho^{2}$ and both charges describe a complete orbit in the same time $T^{(0)}$

$$
T^{(0)}=\frac{2 \pi \rho}{v_{ \pm}}=\frac{2 \pi}{n}=2 \pi \sqrt{\frac{m \rho}{|q| E}}=2 \pi \sqrt{\frac{m \rho^{3}}{|q Q|}}
$$

where it should be noted that the mean motion (i.e. orbital frequency) $n=\sqrt{|q| E / m \rho}$ depends on the charge-to-mass ratio $q / m$.

If we switch on a magnetic field $\boldsymbol{B}=B \boldsymbol{i}_{z}$ orthogonal to the plane of motion, the two charges will experience an additional Lorentz force $\boldsymbol{F}_{L}=(q / c) \boldsymbol{v} \times \boldsymbol{B}$, which for $q>0$ will be antiparallel to the electric field for the counterclockwise moving charge and parallel to the electric field for the clockwise moving charge. Hence the equation of motion for $q>0$ becomes

$$
m \frac{v_{ \pm}^{2}}{\rho}=q E \mp \frac{q}{c} v_{ \pm} B
$$

and therefore

$$
\left(v_{ \pm} \pm \frac{q B}{2 m c} \rho\right)^{2}=\left(n^{2}+\frac{q^{2} B^{2}}{4 m^{2} c^{2}}\right) \rho^{2} .
$$

We assume that the magnetic field is weak; therefore, we can neglect the square of the Larmor frequency $q^{2} B^{2} / 4 m^{2} c^{2}$ in comparison with the square of the orbital frequency $n^{2}$ in equation (4) and obtain $v_{ \pm}=(n \mp q B / 2 m c) \rho$, i.e.

or

$$
\left(\frac{\mathrm{d} \phi}{\mathrm{d} t}\right)_{ \pm}= \pm\left(n \mp \frac{q B}{2 m c}\right)
$$

$$
\mathrm{d} t_{ \pm}= \pm \frac{\mathrm{d} \phi}{n\left(1 \mp \frac{q B}{2 m c n}\right)} \simeq \pm \frac{\mathrm{d} \phi}{n}\left(1 \pm \frac{q B}{2 m c n}\right) .
$$


By integrating equation (6) from 0 to $2 \pi$ for the counterclockwise orbit and $2 \pi$ to 0 for the clockwise orbit, we find the orbital periods of the two charges

$$
T_{ \pm}=\frac{2 \pi}{n}\left(1 \pm \frac{q B}{2 m c n}\right)=T^{(0)} \pm \frac{\pi}{c} \frac{B}{E} \rho
$$

and hence their difference after one revolution

$$
T_{+}-T_{-}=\frac{2 \pi}{c} \frac{B}{E} \rho .
$$

By inspection of equation (7) we see that the magnetic correction to the orbital period is independent of the charge-to-mass ratio of the orbiting electric charges, in contrast to the unperturbed period $T^{(0)}$. Indeed, we could have started section 2 with the less restrictive assumption that the two charges only have the same charge-to-mass ratio.

It is interesting to observe that equation (8) is an exact consequence of equation (3), i.e. the weak-field approximation is unnecessary for the validity of this relation. This circumstance has an analogue in the gravitational case discussed in section 3; that is, equation (17) below turns out to be exact for circular orbits in the equatorial plane of the exterior Kerr spacetime.

Let us now assume that the two charges are far away from the central charge and current distributions so that the magnetic field can be considered to be generated by a magnetic dipole $\boldsymbol{m}=-\mu \boldsymbol{i}_{z}$ of magnitude $\mu=I S / c$, where $S$ is the surface area of the loop enclosed by the current $I$ and, therefore

$$
B=\frac{\mu}{\rho^{3}} \quad E=\frac{|Q|}{\rho^{2}}
$$

which upon inserting into equation (8) yield

$$
T_{+}-T_{-}=\frac{2 \pi}{c} \frac{\mu}{|Q|} .
$$

This time difference depends on both the signs of the charges and on the direction of the magnetic field. Upon exchanging the signs of $Q$ and $q$, i.e. $Q>0$ and $q<0$, the counterclockwise revolving charge will move faster while the clockwise moving charge will move slower. However, as expected by charge symmetry, equation (10) will be unaffected if the signs of the charges and of the magnetic field are reversed simultaneously. Further, we note that the radius of the orbit does not appear in equation (10) and that this time difference can be interpreted as a consequence of the fact that the speed of light has a finite value; indeed, for $c \rightarrow \infty, T_{+}-T_{-} \rightarrow 0$.

The main considerations of this section are related, via the Larmor theorem, to certain interesting phenomena in rotating frames of reference $[8,9]$.

Orbits of charged particles off the equatorial plane are no longer spatially closed in general because their instantaneous planes undergo Larmor precession induced by the magnetic field and it will thus be necessary to define the relevant periods $T_{ \pm}$in terms of azimuthal closure involving a complete loop in the $\phi$ coordinate. For the description of such a configuration it is useful to introduce the local frame attached to the moving particle, where $\boldsymbol{i}_{R}, \boldsymbol{i}_{\mathcal{T}}, \boldsymbol{i}_{N}$ denote its orthogonal unit vectors related to the radial, along-track and cross-track directions, respectively. In fact, $\boldsymbol{i}_{\mathcal{T}}$ denotes the orthogonal direction, in the instantaneous orbital plane, to the radial one; in general, it does not coincide with the direction along the track unless one considers circular orbits. We will return to the case of spherical orbits in section 4 , where the local frame described here is employed along the unperturbed orbit. 


\section{The gravitational scenario}

The electromagnetic scenario previously described is analogous to the following gravitational one: let us consider a central spherically symmetric mass $M$ rotating slowly with its proper angular momentum directed along the $z$-axis, $\boldsymbol{J}=\boldsymbol{J} \boldsymbol{i}_{z}$, of an asymptotically inertial frame $K\{x, y, z\}$ whose $(x, y)$-plane coincides with the equatorial plane of the gravitating source, and a pair of test bodies orbiting along a circular equatorial path in opposite directions. Further, we assume that the radius $\rho$ of the orbit is much larger than the Schwarzschild radius $r_{g}=2 G M / c^{2}$ of the central body; this would apply to an experiment in the field of the Earth, for instance. It is well known that in the weak-field and slow-motion approximation of general relativity the stationary spacetime metric of a rotating 'spherically symmetric' mass-energy distribution generates the so-called gravitoelectric and gravitomagnetic fields [10, 11]

$$
\begin{aligned}
& \boldsymbol{E}_{g}=-\frac{G M}{r^{2}} \boldsymbol{i}_{R} \\
& \boldsymbol{B}_{g}=\nabla \times \boldsymbol{A}_{g}=\frac{2 G}{c}\left[\frac{\boldsymbol{J}-3\left(\boldsymbol{J} \cdot \boldsymbol{i}_{R}\right) \boldsymbol{i}_{R}}{r^{3}}\right]
\end{aligned}
$$

where the gravitomagnetic potential is given by

$$
\boldsymbol{A}_{g}=-\frac{2 G}{c} \frac{\boldsymbol{J} \times \boldsymbol{r}}{r^{3}} .
$$

At this point the calculations follow closely those of the electromagnetic case previously examined in section 2 because the equation of motion of a test body in the 'weak' gravitational field of a general stationary axisymmetric mass-energy distribution is analogous to that of a point charge $q$ acted upon by electric and magnetic fields $\boldsymbol{E}$ and $\boldsymbol{B}$,

$$
m \boldsymbol{a}_{g}=m\left(\boldsymbol{E}_{g}+\frac{\boldsymbol{v}}{c} \times \boldsymbol{B}_{g}\right) .
$$

Therefore, by reasoning as in the electromagnetic case for circular orbits with zero inclination (section 2), we obtain

$$
T_{+}-T_{-}=\frac{2 \pi}{c} \frac{B_{g}}{E_{g}} \rho .
$$

For equatorial circular orbits, equation (12) immediately yields

$$
\boldsymbol{B}_{g}=\frac{2 G}{c} \frac{J}{\rho^{3}} \boldsymbol{i}_{z}
$$

By inserting $E_{g}$ and $B_{g}$ from equations (11) and (16) into equation (15) we obtain the wellknown expression

$$
T_{+}-T_{-}=4 \pi \frac{J}{c^{2} M} .
$$

It is an interesting feature of equation (17) that the mass moving in the same sense of rotation as the central mass moves slower than the mass moving in the opposite direction. If we reversed the sense of rotation of the central gravitating source, the clockwise moving test mass would be slower. In this way the sense of rotation of the central mass is no longer a matter of convention but could be related to a physical phenomenon, i.e. the mass loop moving slower. Also in this case, in the limit $c \rightarrow \infty, T_{+}-T_{-} \rightarrow 0$.

An interesting feature of gravitoelectromagnetism is the gravitational Larmor theorem [12] according to which gravitoelectric and gravitomagnetic fields are locally equivalent to translational and rotational accelerations of an observer in Minkowski spacetime. The gravitational Larmor theorem is therefore in effect Einstein's principle of equivalence in 


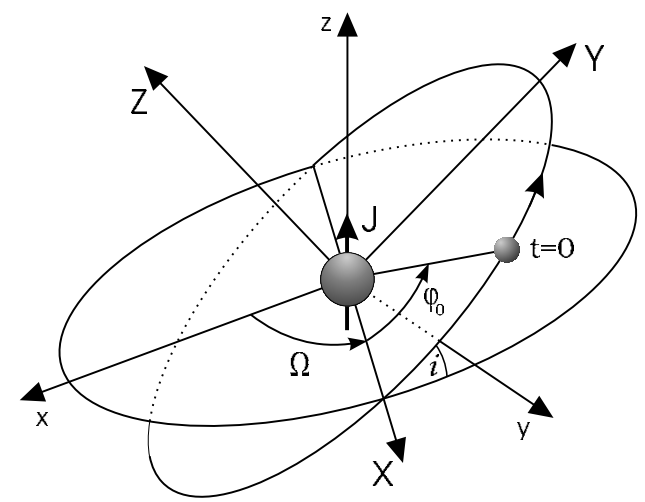

Figure 1. Schematic diagram of the unperturbed circular trajectory for the general spherical orbit. In practice, the instantaneous orbital plane at the instant the observations begin, i.e. $t=0$, can be taken to be the plane of the unperturbed orbit.

the gravitoelectromagnetic context. Note that in equation (17) the gravitoelectric Keplerian periods $T^{(0)}=2 \pi / n=\sqrt{G M / \rho^{3}}$ cancel out, no matter what the masses of the orbiting particles are, in accordance with the equivalence principle and in contrast to the electromagnetic case, where the unperturbed periods depend on the charge-to-mass ratio of the particles.

Finally, it is worth noting that the gravitomagnetic correction to the unperturbed Keplerian period is independent of the radius of the orbit, a feature also common to the electrodynamic case. Moreover, the Newtonian constant $G$ does not appear in equation (17) this fact may account for the unexpectedly large value of the time shift in the field of the Earth which amounts to about $10^{-7} \mathrm{~s}$. In some sense, this classical effect is a gravitomagnetic analogue of the topological Aharonov-Bohm effect. It turns out that if we deal with the proper periods of the test bodies, i.e. the periods according to comoving clocks, then the result is the same as in equation (17) up to terms of order $c^{-4}$ that depend on $G$ and $\rho$.

\section{Arbitrary inclination: inertial azimuthal closure}

If the orbital plane has an arbitrary inclination $i$ to the equatorial plane of $K\{x, y, z\}$, a similar reasoning as in section 2 holds: the orbital plane undergoes Lense-Thirring precession, which is the gravitational analogue of the magnetic Larmor precession. Let us imagine that in the absence of the gravitomagnetic field, the orbit is a circle of radius $r$ in a fixed plane that is inclined with respect to the equatorial $(x, y)$-plane by the inclination angle $i$; that is, the normal to the orbital plane is tilted away from the $z$-axis by the angle $i$. Moreover, the longitude of the ascending node is given by the azimuthal angle $\Omega$, as in figure 1. Once the gravitomagnetic field is 'turned on,' the orbit will twist out of this fixed plane.

In order to derive analytically the time $T$ needed to pass from $\phi_{0}$ at $t_{0}=0$ to $\phi_{0}+2 \pi$ at $t_{0}+T$ in the equatorial plane of the inertial observer we will use the following reference frames: the asymptotically inertial frame $K\{x, y, z\}$ previously defined and a frame $K^{\prime}\{X, Y, Z\}$ with the $Z$-axis directed along the orbital angular momentum $\boldsymbol{L}$ of the unperturbed test body, where the $(X, Y)$-plane coincides with the unperturbed orbital plane of the test particle and the $X$-axis is directed along the line of nodes. $K\{x, y, z\}$ and $K^{\prime}\{X, Y, Z\}$ have the same origin located at the centre- of-mass of the central body. The transformation matrix $\boldsymbol{R}_{x X}$ for the change of coordinates from $K^{\prime}\{X, Y, Z\}$ to $K\{x, y, z\}$ is given by 


$$
\boldsymbol{R}_{x X}=\left(\begin{array}{ccc}
\cos \Omega & -\sin \Omega \cos i & \sin \Omega \sin i \\
\sin \Omega & \cos \Omega \cos i & -\cos \Omega \sin i \\
0 & \sin i & \cos i
\end{array}\right)
$$

Our calculation for the azimuthal period $T$ will be valid for a counterclockwise orbit as in figure 1; however, it is clear from the symmetry of the configuration that the result for the clockwise case $\left(T_{-}\right)$can be simply obtained from our result by reversing the sign of the perturbation term in $T_{+}$. In the asymptotically inertial frame $K^{\prime}\{X, Y, Z\}$, let us choose cylindrical coordinates $\{\rho, \varphi, Z\}$ and write equation (14) in these coordinates. The unperturbed orbit is given by $\rho \equiv r=$ constant, $Z=0$ and $\varphi=\varphi_{0}+n t$ (with $n=\sqrt{G M / \rho^{3}}$ ). Since we will consider the gravitomagnetic acceleration $c^{-1} \boldsymbol{v} \times \boldsymbol{B}_{g}$ as a small perturbation of the main gravitoelectric monopole term $\boldsymbol{E}_{g}$, we evaluate the disturbing acceleration with respect to the unperturbed orbit. By using equations (11) and (12), equation (14) can be written as

$$
\begin{aligned}
& a_{\rho}=\ddot{\rho}-\rho \dot{\varphi}^{2}=-\frac{G M}{\rho^{2}}+2 \frac{G J}{c^{2} \rho^{2}} n \cos i \\
& a_{\varphi}=\rho \ddot{\varphi}+2 \dot{\rho} \dot{\varphi}=0 \\
& a_{Z}=\ddot{Z}=-n^{2} Z+4 \frac{G J}{c^{2} \rho^{2}} n \sin i \sin \left(\varphi_{0}+n t\right) .
\end{aligned}
$$

Here, the radial and along-track components of the acceleration are given by $a_{\rho}=$ $\ddot{X} \cos \varphi+\ddot{Y} \sin \varphi$ and $a_{\varphi}=-\ddot{X} \sin \varphi+\ddot{Y} \cos \varphi$, respectively. Note that in equation (21) we retain $Z$, because, due to the gravitomagnetic non-central acceleration, the motion is no longer confined to a plane and, therefore, $Z$ will be proportional to the gravitomagnetic perturbation and treated to first order.

For a spherical orbit $\rho$ remains constant to first order; therefore, from equation (19) we obtain

$$
\varphi=\varphi_{0}+n t-\frac{G J}{c^{2} \rho^{3}} t \cos i \equiv \hat{\varphi}+\delta \varphi
$$

where $\hat{\varphi}=\varphi_{0}+n t$. It follows from the linear dependence of $\varphi$ on $t$ in equation (22) that equation (20) is satisfied. The solution of equation (21) reads

$$
Z=-2 \frac{G J}{c^{2} \rho^{2}} t \sin i \cos \left(\varphi_{0}+n t\right)+k_{1} \sin n t+k_{2} \cos n t
$$

where $k_{1}$ and $k_{2}$ are constants of integration. If we assume that at $t_{0}=0$ the perturbed orbit agrees with the unperturbed orbit, i.e. $Z(0)=0$, then $k_{2}=0$ and we will treat $k_{1}$ to first order of the perturbation in what follows. Let us write the general solution of equations (19)-(21) as $X=\rho \cos \varphi(t)$ and $Y=\rho \sin \varphi(t)$ together with equation (23). By using $\cos (\hat{\varphi}+\delta \varphi) \simeq$ $\cos \hat{\varphi}-\delta \varphi \sin \hat{\varphi}$ and $\sin (\hat{\varphi}+\delta \varphi) \simeq \sin \hat{\varphi}+\delta \varphi \cos \hat{\varphi}$, we find

$$
\begin{aligned}
& X=\rho \cos \hat{\varphi}+\frac{G J}{c^{2} \rho^{2}} t \cos i \sin \hat{\varphi} \\
& Y=\rho \sin \hat{\varphi}-\frac{G J}{c^{2} \rho^{2}} t \cos i \cos \hat{\varphi} \\
& Z=-2 \frac{G J}{c^{2} \rho^{2}} t \sin i \cos \hat{\varphi}+k_{1} \sin n t
\end{aligned}
$$


From these equations we obtain the solution in $K\{x, y, z\}$ by means of the transformation (18) $x=\rho(\cos \Omega \cos \hat{\varphi}-\cos i \sin \Omega \sin \hat{\varphi})+k_{1} \sin i \sin \Omega \sin n t$

$$
+\frac{G J}{c^{2} \rho^{2}} t\left[\cos i \cos \Omega \sin \hat{\varphi}+\left(\cos ^{2} i-2 \sin ^{2} i\right) \sin \Omega \cos \hat{\varphi}\right]
$$

$y=\rho(\sin \Omega \cos \hat{\varphi}+\cos i \cos \Omega \sin \hat{\varphi})-k_{1} \sin i \cos \Omega \sin n t$

$$
+\frac{G J}{c^{2} \rho^{2}} t\left[\cos i \sin \Omega \sin \hat{\varphi}-\left(\cos ^{2} i-2 \sin ^{2} i\right) \cos \Omega \cos \hat{\varphi}\right]
$$

$z=\rho \sin i \sin \hat{\varphi}+k_{1} \cos i \sin n t-\frac{3}{2} \frac{G J}{c^{2} \rho^{2}} t \sin 2 i \cos \hat{\varphi}$.

The temporal behaviour of the azimuthal angle $\phi$ can be obtained via

$$
\tan \phi=\frac{y(t)}{x(t)}
$$

and the time $T$ needed to pass from $\phi_{0}$ at $t_{0}=0$ to $\phi_{0}+2 \pi$ at $t_{0}+T$ follows upon expanding the relation

$$
\tan \phi_{0}=\tan \left(\phi_{0}+2 \pi\right)
$$

where

and

$$
\tan \phi_{0}=\frac{y(0)}{x(0)}=\frac{\sin \Omega \cos \varphi_{0}+\cos i \cos \Omega \sin \varphi_{0}}{\cos \Omega \cos \varphi_{0}-\cos i \sin \Omega \sin \varphi_{0}}
$$

$$
\tan \left(\phi_{0}+2 \pi\right)=\frac{y(T)}{x(T)}
$$

Since the deviation from the unperturbed Kepler period $T^{(0)}=2 \pi / n$ will be small, let us write

$$
T=\frac{2 \pi}{n}(1+\epsilon)
$$

with $\epsilon \ll 1$ and further

$\sin n T=\sin (2 \pi+2 \pi \epsilon) \simeq 2 \pi \epsilon$

$\cos \hat{\varphi}(T)=\cos \left(\varphi_{0}+n T\right)=\cos \left(\varphi_{0}+2 \pi+2 \pi \epsilon\right) \simeq \cos \varphi_{0}-2 \pi \epsilon \sin \varphi_{0}$

$\sin \hat{\varphi}(T)=\sin \left(\varphi_{0}+n T\right)=\sin \left(\varphi_{0}+2 \pi+2 \pi \epsilon\right) \simeq \sin \varphi_{0}+2 \pi \epsilon \cos \varphi_{0}$.

Therefore, in the calculations for $x(T)$ and $y(T)$, terms proportional to $k_{1} \sin n T$, due to equation (35), will be of second order and will be neglected. Hence we find

$$
\begin{array}{r}
x(T)=\rho\left(\cos \Omega \cos \varphi_{0}-\cos i \sin \Omega \sin \varphi_{0}\right)-2 \pi \epsilon \rho\left(\cos \Omega \sin \varphi_{0}+\cos i \sin \Omega \cos \varphi_{0}\right) \\
+2 \pi \frac{G J}{c^{2} \rho^{2}} \frac{1}{n}\left[\cos i \cos \Omega \sin \varphi_{0}+\left(\cos ^{2} i-2 \sin ^{2} i\right) \sin \Omega \cos \varphi_{0}\right] \\
y(T)=\rho\left(\sin \Omega \cos \varphi_{0}+\cos i \cos \Omega \sin \varphi_{0}\right)-2 \pi \epsilon \rho\left(\sin \Omega \sin \varphi_{0}-\cos i \cos \Omega \cos \varphi_{0}\right) \\
+2 \pi \frac{G J}{c^{2} \rho^{2}} \frac{1}{n}\left[\cos i \sin \Omega \sin \varphi_{0}-\left(\cos ^{2} i-2 \sin ^{2} i\right) \cos \Omega \cos \varphi_{0}\right] .
\end{array}
$$

Using equations (31)-(33) and equations (38) and (39), we find after some algebra

$$
\epsilon=\frac{G J}{c^{2} \rho^{3}} \frac{\cos i}{n}\left(1-2 \tan ^{2} i \cos ^{2} \varphi_{0}\right)
$$

As expected, $\epsilon$ is proportional to $J$ and vanishes for a non-rotating source. By means of equation (34) it finally follows that

$$
T_{ \pm}=\frac{2 \pi}{n}\left[1 \pm \frac{G J}{c^{2} \rho^{3}} \frac{\cos i}{n}\left(1-2 \tan ^{2} i \cos ^{2} \varphi_{0}\right)\right]
$$




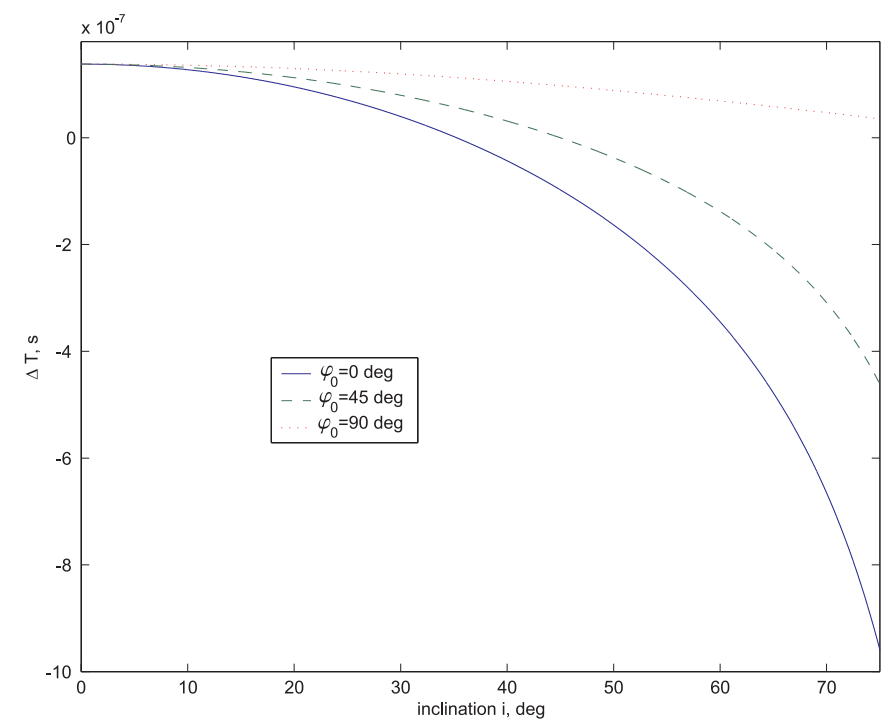

Figure 2. Plot of $\Delta T=T_{+}-T_{-}$, given in equation (42), in seconds versus the orbital inclination $i$ in degrees for a satellite in a spherical orbit around the Earth. For inclined orbits, the three curves illustrate the dependence of the gravitomagnetic clock effect on $\varphi_{0}$, which is the angular position of the satellite along its circular orbit at $t=0$ measured from the line of the ascending node (cf figure 1).

or

$$
T_{+}-T_{-}=4 \pi \frac{J \cos i}{c^{2} M}\left(1-2 \tan ^{2} i \cos ^{2} \varphi_{0}\right)
$$

Let us note that for $i=0$, we recover the result of section 3 . On the other hand, $\epsilon$ diverges for $i=\pi / 2$. It follows from general relativity that for a geodesic (spherical) polar orbit, the clock effect disappears since the angular momentum vector of the source in effect lies in the orbital plane. The orbital period is then simply given by the gravitoelectric Keplerian period. On the other hand, the period for azimuthal closure is given by $2 \pi\left(2 G J / c^{2} \rho^{3}\right)^{-1}$, which is very long compared to the Keplerian period [13]. This circumstance is reflected in our first-order perturbative result given by equation (41): for $i \rightarrow \pi / 2, T_{ \pm} \rightarrow \infty$. Therefore, in equation (41), the inclination angle $i$ must be sufficiently less than $\pi / 2$ such that the perturbative treatment in section 4 remains valid.

An important feature of equation (41) is that when $i \neq 0, T_{ \pm}$depends upon $\varphi_{0}$, i.e. the clock effect depends in general on the position of the mass $m$ along the orbit at $t=0$. This dependence of the clock effect on where the mass $m$ is along the orbit when the timing observations begin is illustrated in figure 2 and could be helpful in the detection of this effect.

Up to now we have assumed that when unperturbed the two satellites orbit along opposite directions in the same plane with arbitrary inclination $i$. Let us now consider the case of two masses $m_{+}$and $m_{-}$having the same distance from the centre but moving in different orbital planes, say $0<i_{+}<\pi / 2$ and $\pi / 2<i_{-}<\pi$, respectively. Following the same reasoning as before, their orbital periods, as viewed by a static, asymptotically inertial observer and after choosing $t_{0}$ suitably so that $\varphi_{0}=\pi / 2$, can be written as

$$
T_{-}=T^{(0)}+2 \pi \frac{J}{c^{2} M} \cos i_{+}
$$




$$
T_{-}=T^{(0)}-2 \pi \frac{J}{c^{2} M} \cos i_{-} .
$$

From these expressions, we immediately find

$$
\begin{aligned}
& T_{-}-T_{-}=2 \pi \frac{J}{c^{2} M}\left(\cos i_{+}+\cos i_{-}\right) \\
& T_{-}+T_{-}=2 T^{(0)}+2 \pi \frac{J}{c^{2} M}\left(\cos i_{+}-\cos i_{-}\right) .
\end{aligned}
$$

From equation (46) it is seen that the sum of the orbital periods of the two point masses will also show a gravitomagnetic contribution provided the inclinations of the two satellites are different. Note that equation (45) reduces correctly to equation (42) if the two orbital planes coincide, while in equation (46) the gravitomagnetic contribution vanishes. Moreover, a very interesting feature arises for supplementary inclinations of the two satellites, i.e. $i_{+}+i_{-}=180^{\circ}$; indeed, in this case equations (45) and (46) become

$$
\begin{aligned}
& T_{-}-T_{-}=0 \\
& T_{-}+T_{-}=2 T^{(0)}+4 \pi \frac{J}{c^{2} M} \cos i_{+} .
\end{aligned}
$$

One of the most striking implications of equation (48) is that the LARES mission [14], originally proposed to detect the Lense-Thirring drag of the orbital plane, could also be used to detect the gravitomagnetic contribution to the sum of the orbital periods. However, due to the present uncertainty of the value of $G M_{\oplus}, \delta\left(G M_{\oplus}\right)=8 \times 10^{11} \mathrm{~cm}^{3} \mathrm{~s}^{-2}$ [15], the error in the unperturbed Keplerian period of the LAGEOS satellite is larger than the effect to be measured

$$
\begin{aligned}
& \delta T^{(0)}=1.52 \times 10^{-5} \mathrm{~s} \\
& 4 \pi \frac{J}{c^{2} M} \cos i_{+}=4.71 \times 10^{-8} \mathrm{~s} .
\end{aligned}
$$

Therefore, an improvement of our knowledge of $G M_{\oplus}$ would be necessary before any observables involving the sum of the unperturbed orbital periods and of the gravitomagnetic corrections may become detectable.

Finally, these results suggest that the gravitomagnetic clock effect may be enhanced by considering suitable constellations of satellites orbiting the Earth.

\section{Conclusions}

Exploiting the formal analogy between the law of motion of a charged particle acted upon by an electromagnetic field and the weak-field and slow-motion approximation of general relativity ('gravitoelectromagnetism'), it has been possible to derive the gravitomagnetic clock effect for a couple of point masses following spherical orbits in space in a simple fashion. General expressions for arbitrary values of inclination angle to the equatorial plane are given for these spherical orbits. These results are obtained by neglecting terms of $\mathcal{O}\left(c^{-4}\right)$; in this way, equation (42) is equally valid for proper periods of co-moving clocks. If the two satellites orbit in planes with different inclinations, the sum of their orbital periods also exhibits a gravitomagnetic part.

It is worthwhile to compare our main result equation (42) with the corresponding general relativistic expression (see [2] p. 143, equations (36) and (37))

$$
T_{+}-T_{-}=4 \pi \frac{J}{c^{2} M} \lambda^{\prime} \cos \alpha
$$


where

$$
\lambda^{\prime}=\lambda-3 \Phi_{0} \Gamma_{0}=\Gamma_{0}-2 \Gamma_{0}^{-1} \tan ^{2} \alpha \cos ^{2} \eta_{0}-3 \Phi_{0} \Gamma_{0} .
$$

Here, $\alpha$ and $\eta_{0}$ must be replaced by $i$ and $\varphi_{0}$, respectively, and $\Gamma_{0} \rightarrow 1, \Phi_{0} \rightarrow 0$ provided that terms of $\mathcal{O}\left(c^{-4}\right)$ are neglected. For the proper periods of co-moving clocks an equation similar to equation (51) holds, except that $\lambda^{\prime}$ must be replaced by $\lambda$. Note that these results are obtained in a perturbative way and hold only for $\alpha$ sufficiently different from $\pi / 2$. Thus, our approach gives the same result for $T_{+}-T_{-}$as general relativity once terms of order $c^{-4}$ are neglected.

As it is for all general relativistic effects, the observation of the gravitomagnetic clock effect is a tremendously difficult undertaking as well. This becomes immediately clear by noting that for a near-Earth orbit $\left(T \sim 10^{4} \mathrm{~s}\right)$ a time variation of $\sim 100 \mathrm{~ns}$ can be caused equally well by a radial or azimuthal deviation of $\sim 0.1 \mathrm{~mm}$ from the ideal orbit and therefore, all forces that may produce accelerations larger than $\sim 10^{-12} \mathrm{~m} \mathrm{~s}^{-2}$ must be taken into account. The empirical verification of the clock effect essentially faces two problems: (a) to measure with the utmost precision the actual position of the satellites and (b) to model with extreme accuracy all perturbing forces which will influence the period of the satellites. For a single orbit, this goal is certainly unattainable, but it may become feasible after a sufficiently long time of observation due to the accumulative character of the clock effect. While the present satellite-to-satellite tracking techniques allow the determination of an orbit with an accuracy of $\sim 1 \mathrm{~cm}$ so that a minimum of $\sim 1000$ revolutions will be needed for the clock effect to become detectable, the consideration of the perturbing forces at the required level is quite demanding. Non-gravitational perturbations may be overcome by means of modern drag-free technology; however, the correct determination of all gravitational effects is limited by the accuracy of the respective Earth gravity field models that are currently available. Preliminary results suggest that it is in particular the uncertainty in the even zonal harmonics of the spherical expansion of the terrestrial gravitational field [7] as well as the zonal tidal perturbations [4] that presently inhibits the successful realization of the clock experiment. It should be mentioned, however, that upcoming geodetic space missions (especially GRACE and GOCE) are expected to improve the accuracy of the gravity field of the Earth significantly and may then allow the observation of the gravitomagnetic clock effect within a few per cent accuracy.

\section{Acknowledgment}

LI is grateful to L Guerriero and I Ciufolini for their support and encouragement.

\section{References}

[1] Cohen J M and Mashhoon B 1993 Phys. Lett. A 181353

[2] Mashhoon B, Gronwald F and Theiss D S 1999 Ann. Phys., Lpz. 8135

[3] Gronwald F, Gruber E, Lichtenegger H I M and Puntigam R A 1997 ESA SP-420 29

[4] Iorio L 2001 Int. J. Mod. Phys. D 10465

[5] Iorio L 2001 Class. Quantum Grav. 184303

[6] Lichtenegger H I M, Gronwald F and Mashhoon B 2000 Adv. Space Res. 251255

[7] Lichtenegger H I M, Hausleitner W, Gronwald F and Mashhoon B 2001 Preprint gr-qc/0101089

[8] Opat G I 1990 Am.J. Phys. 581173

[9] Opat G I 1991 Am. J. Phys. 59822

[10] Ciufolini I and Wheeler J A 1995 Gravitation and Inertia (New York: Princeton University Press) p 498 
[11] Mashhoon B, Gronwald F and Lichtenegger H I M 2001 Gyros, Clocks, Interferometers .... : Testing Relativistic Gravity in Space (Lecture Notes in Physics vol 562) ed C Lämmerzahl, C W F Everitt and F W Hehl (Berlin: Springer) pp 83-108

[12] Mashhoon B 1993 Phys. Lett. A 173347

[13] Mashhoon B and Theiss D S 1982 Phys. Rev. Lett. 491542

[14] Ciufolini I 1986 Phys. Rev. Lett. 56278

[15] McCarthy D D 1996 IERS Technical Note vol 21 (US Naval Observatory) p 95 